\title{
Chronic pain and quality of life after transinguinal preperitoneal (TIPP) inguinal hernia repair: a few tips on TIPP
}

\author{
D. C. Chen • P. K. Amid
}

Received: 23 June 2013/Accepted: 6 July 2013/Published online: 23 July 2013

(C) Springer-Verlag France 2013

Keywords Chronic pain after hernia repair .

Inguinodynia $\cdot$ Tipp $\cdot$ Preperitoneal

Dear Editors,

It was with great interest that we read the manuscript "Chronic Pain and Quality of Life after Transinguinal Preperitoneal (TIPP) Inguinal Hernia Repair". The focus on chronic pain and quality of life as the most relevant clinical outcomes of inguinal hernia repair is a testament to the success of modern techniques and the use of prosthetic materials which have dramatically reduced recurrence rates. Inguinodynia, however, has been a problem since the inception of herniorrhaphies and long-preceded meshbased techniques $[1,2]$. In this article, the authors describe their experience with a modified transinguinal preperitoneal (TIPP) procedure using a Polysoft ring patch. It is the largest series examining subjectively reported QOL and pain using this technique and helps to confirm the efficacy and excellent outcomes of TIPP as a predictable, safe, costeffective, option for elective inguinal herniorrhaphy.

Advocates of TIPP have touted its benefits over laparoscopic TEP repair in regards to predictability, cost, technical simplicity, and safety [3, 4]. Similarly, TIPP has been compared to traditional anterior Lichtenstein repair with Koning's group (TULIP trial) reporting a significant decrease in chronic pain at 1 year (3.5 versus $12.9 \%$ ) [3].

D. C. Chen $(\bowtie) \cdot$ P. K. Amid

Division of General Surgery, Lichtenstein Amid Hernia Clinic at UCLA, David Geffen School of Medicine at UCLA, 1304 15th Street Suite 213, Santa Monica, CA 90404, USA

e-mail: dcchen@mednet.ucla.edu

P. K. Amid

e-mail: pamid@mednet.ucla.edu
While TIPP has proven to be a viable and effective preperitoneal technique, to answer the question of "Is TIPP top?" requires a more critical assessment $[3,4]$. The initial retrospective results from the TULIP group did not identify a difference in the rate of chronic pain (4.4\% TIPP versus $4.1 \%$ Lichtenstein) [4]. Furthermore, while the results of the randomized, prospective TULIP trial demonstrated a significant difference, a $12.9 \%$ rate of chronic pain for a Lichtenstein repair is far from enviable and far exceeds their prior results of $4.1 \%$ or the ideal rate of $0.5 \%$ achievable with three nerve identification and proper nerve handling [3-5].

In this study, the authors report excellent outcomes and commendable QOL data but VAS scores were obtained only to postoperative day 30 . The follow-up period is too short to draw any broader comparisons to established methods such as Lichtenstein and TEP that have well established, reproducible long-term data on outcomes including QOL, recurrence, and chronic pain. While there are no long-term VAS scores to compare, in Group 3 of the long-term QOL data, about $25 \%$ (128 of 531) of patients had pain. Of these, $14.5 \%$ (77 of 531) had moderate to severe pain while $18 \%$ (96 of 531) reported pain several times per week or greater. These data are in line with most other techniques but would not be considered superior. The more mature view of hernia repair is that there are several good options for anterior, posterior, open, and laparoscopic repair with TIPP being one of these. Similarly, with regards to pain, rather than superiority of any one technique, the reality is more "pick your poison" as each technique has a different profile of potential causes of inguinodynia. Anterior repairs may have higher reported rates of pain but are easier to remediate [6]. Laparoscopic repairs have excellent overall outcomes, but pain and orchialgia are technically more challenging to address [7-9]. The 
individual surgeon would be best suited to perfect a preferred technique and master different operative alternatives for different clinical circumstances in order to decrease personal rates of pain and improve outcomes.

With regards to the technical considerations and etiology of pain associated with TIPP repair, we would like to clarify some misperceptions with regard to the neuroanatomy of the preperitoneal space. The important developments by Nyhus, Read, Stoppa, Wantz, Rives, Shumpelick, and others have helped to define and utilize the preperitoneal anatomy for effective hernia repairs. Laparoscopic TEP and TAPP have made this compartmental anatomy ubiquitous for most hernia surgeons. However, in depth understanding of the neuroanatomy of this compartment is needed to prevent neuralgia, orchialgia, dysejaculation, recurrence, and meshoma. Read, Skandalakis, and Mirilas have helped to delineate the surgical anatomy of the preperitoneal space confirming the presence of two compartments behind the transversalis fascia separated by a membranous layer $[10,11]$. The visceral compartment medially contains the bladder, ureter, and prostate and laterally contains the vas deferens ensheathed by this membranous layer. The parietal compartment contains the genitofemoral trunk and its genital and femoral branches. The lateral femoral cutaneous, ilioinguinal, and iliohypogastric nerves also travel in this compartment but are usually cephalad or lateral to most preperitoneal repairs. Unlike the nerves in the inguinal canal that are protected by an investing fascia (investing fascia of the internal oblique for the ilioinguinal and iliohypogastric nerves; deep cremasteric fascia for the inguinal segment of the genital branch), the nerves in this compartment are naked $[6,8$, 11]. Mesh placed in the parietal compartment during laparoscopic and open preperitoneal herniorrhaphy may produce chronic neuropathic inguinodynia or orchialgia [7]. Remedial surgery is more challenging than with anterior repair and requires proximal access to these nerves via laparoscopic retroperitoneal triple neurectomy with neuropathic inguinodynia or proximal neurolysis of the paravasal nerve fibers with orchialgia [9].

There are some inherent risks associated with TIPP because it requires entering the inguinal canal for access to the internal ring and/or the transversalis fascia. Surgical manipulation of both inguinal canal and preperitoneal space can potentially injure the ilioinguinal nerve, iliohypogastric nerve, and the inguinal segment of the genital branch of the genitofemoral nerve within the inguinal canal. In addition, preperitoneal dissection can injure the main trunk of the genitofemoral nerve, its genital and femoral branches, the vas deferens, and inferior epigastric vessels within the preperitoneal space $[11,12]$. The authors clearly mention the importance of nerve identification [5]. However, they have very low rates of nerve identification with the ilioinguinal nerve not seen in $24.3 \%$ of patients, the iliohypogastric nerve in $84.9 \%$, and the genital nerve not identified in $78.3 \%$. Although this technique is less likely to injure the iliohypogastric nerve, three nerve identification is recommended in all anterior approaches [5].

The claims that the nerves are not in contact with mesh are inaccurate. Mesh placed in the preperitoneal space (TEP, TAPP, plug, TIPP, PHS, Kugel) laparoscopically or transinguinally abuts the genital branch and GFN, and can reach the LFC depending on the location and size of the prosthesis and the amount of dissection performed [11]. As described, the nerves in this space do not have an investing fascia and neuropathy from direct contact is a known cause for inguinodynia. The authors claim that the genital nerve is not at risk because it is separated from the mesh by the "internal spermatic vessels and the retroparietal spermatic sheath described by Stoppa". This layer is actually the membranous layer that ensheathes the vas deferens and gonadal vessels within the parietal compartment [11]. While a membranous layer of the extraperitoneal fascia clearly exists dividing the preperitoneal space into a visceral and parietal compartment, Mirales et al. [11] have clearly demonstrated that this layer is located behind the nerves thus it cannot protect the genital and femoral branches of the genitofemoral nerves in the TIPP operation. In this regard, placement of mesh in the visceral compartment typically accessed in laparoscopic TEP and TAPP is theoretically less likely to cause genitofemoral causalgia.

Another important anatomic consideration is that the preperitoneal dissection is functionally blind and cannot be compared to laparoscopic TEP and TAPP where all structures can be visualized including nerves and vascular structures as well as the separation between the visceral and parietal compartments. This is painfully apparent in a TEP operation when a separating balloon dissects the parietal compartment leaving the epigastric vessels down. The authors state that parietalization in TIPP is not a blind procedure as direct visualization is possible through the internal ring or direct defect. Through a $3.5-\mathrm{cm}$ incision and with retraction of the deep ring, they can attain "sufficient exposure to the preperitoneal structures allowing complete dissection under permanent visual control." TIPP reportedly can lead to major preperitoneal bleeding and hematoma due to compromise of the epigastric vessels. Other reported complications associated with a preperitoneal approach include dysejaculation, mesh migration, and urinary complaints. Many of these significant complications will present later than the endpoints of this study [12]. The authors assert that "TIPP is actually a TEP performed via an external mini invasive approach". Just as in all anterior approaches through a small incision, lack of visualization is the expected cost as compared to 
laparoendoscopic approaches. This does not make it inferior (as Plug, PHS, Kugel and other preperitoneal repairs follow this same approach) but the limitation should be acknowledged.

Over the past 30 years, thousands of patients evaluated for inguinodynia at the Lichtenstein Amid Hernia Clinic have presented after all methods and variations of hernia repair-anterior/posterior, tissue/prosthetic, open/laparoscopic. While there are clear technical considerations with each operation that can predispose patients to favorable or unfavorable outcomes, there is no definite superiority of one technique over another in the incidence and prevention of pain [5]. As seen with the authors' reported large experience and excellent outcomes with TIPP, centers dedicated to refining a specific technique will likely demonstrate and publish excellent outcomes but the true incidence of pain in general clinical practice remains disturbingly high. For the individual patient that develops chronic pain, the choice of any specific technique was the wrong one. Thorough understanding of each technique and the specific risks associated with a specific operation is crucial. The commonality is that in-depth understanding of neuroanatomy will help to improve the patient outcomes as prevention is far more effective than treatment of chronic pain [5].

Conflict of interest D.C. Chen declares no conflict of interest. P.K. Amid declares no conflict of interest.

\section{References}

1. Magee RK (1942) Genitofemoral causalgia: (a new syndrome). Can Med Assoc J 46(4):326-329
2. Lichtenstein IL, Shulman AG, Amid PK, Montllor MM (1988) Cause and prevention of postherniorrhaphy neuralgia: a proposed protocol for treatment. Am J Surg 155(6):786-790

3. Koning GG, Keus F, Koeslag L, Cheung CL, Avçi M, van Laarhoven CJ, Vriens PW (2012) Randomized clinical trial of chronic pain after the transinguinal preperitoneal technique compared with Lichtenstein's method for inguinal hernia repair. Br J Surg 99(10):1365-1373

4. Koning GG, Koole D, de Jongh MA, de Schipper JP, Verhofstad MH, Oostvogel HJ, Vriens PW (2011) The transinguinal preperitoneal hernia correction vs Lichtenstein's technique; is TIPP top? Hernia 15(1):19-22

5. Alfieri S, Amid PK, Campanelli G, Izard G, Kehlet H, Wijsmuller AR, Di Miceli D, Doglietto GB (2011) International guidelines for prevention and management of post-operative chronic pain following inguinal hernia surgery. Hernia 15(3):239-249

6. Amid PK, Hiatt JR (2007) New understanding of the causes and surgical treatment of postherniorrhaphy inguinodynia and orchalgia. J Am Coll Surg 205(2):381-385

7. Amid PK, Chen DC (2011) Surgical treatment of chronic groin and testicular pain after laparoscopic and open preperitoneal inguinal hernia repair. J Am Coll Surg 213:531-536

8. Amid PK, Hiatt JR (2008) Surgical anatomy of the preperitoneal space. J Am Coll Surg 207(2):295

9. Chen DC, Hiatt JR, Amid PK (2013) Operative management of refractory neuropathic inguinodynia by a laparoscopic retroperitoneal approach. JAMA Surg (in press)

10. Read RC (1992) Cooper's posterior lamina of the transversalis fascia. Surg Gynecol Obstet 174:426-434

11. Mirilas P, Mentessidou A, Skandalakis JE (2008) Secondary internal inguinal ring and associated surgical planes: surgical anatomy, embryology, applications. J Am Coll Surg 206(3): 561-570

12. Amid PK (2005) Groin hernia repair: open techniques. World J Surg 29:1046-1051 